\title{
Mechanisms of Heavy Ion-Induced Single Event Burnout in 4H-SiC Power MOSFETs
}

Joseph A. McPherson ${ }^{1}$, Collin Hitchcock ${ }^{1}$, T. Paul Chow ${ }^{1}$, Wei $\mathrm{Ji}^{1^{*}}$, Andrew A. Woodworth ${ }^{2}$

${ }^{1}$ Rensselaer Polytechnic Institute, Troy, New York, U.S.A.; ${ }^{2}$ NASA Glenn Research Center, Cleveland, Ohio, U.S.A.

*Corresponding Author : jiw2@rpi.edu, +1-518-276-6602

\section{Introduction}

Silicon Carbide (SiC) power MOSFETs are capable of operating at higher temperatures, voltages, and switching frequencies than silicon. This makes SiC desirable to NASA for future space missions and electric aircraft propulsion technologies. However, experimental tests have shown that $\mathrm{SiC}$ devices are susceptible to single event effects, especially single event burnout (SEB) caused by heavy ions. SEB can occur at applied voltages of less than half the device rating.

This work uses 3-dimensional electrothermal device simulations to explore the mechanisms of SEB in MOSFETs and proposes new designs that can survive SEB at higher blocking voltages.

\section{Baseline Model}

-Commercially available power MOSFET rated for $1200 \mathrm{~V}$ with a specific on-resistance (Ron,sp) of $3.68 \mathrm{~m} \Omega-\mathrm{cm}^{-2}$

- $10 \mu \mathrm{m}$ thick epitaxial layer at doped n-type $6 \times 10^{15} \mathrm{~cm}-3$ on a $40 \mu \mathrm{m}$ $n+$ substrate
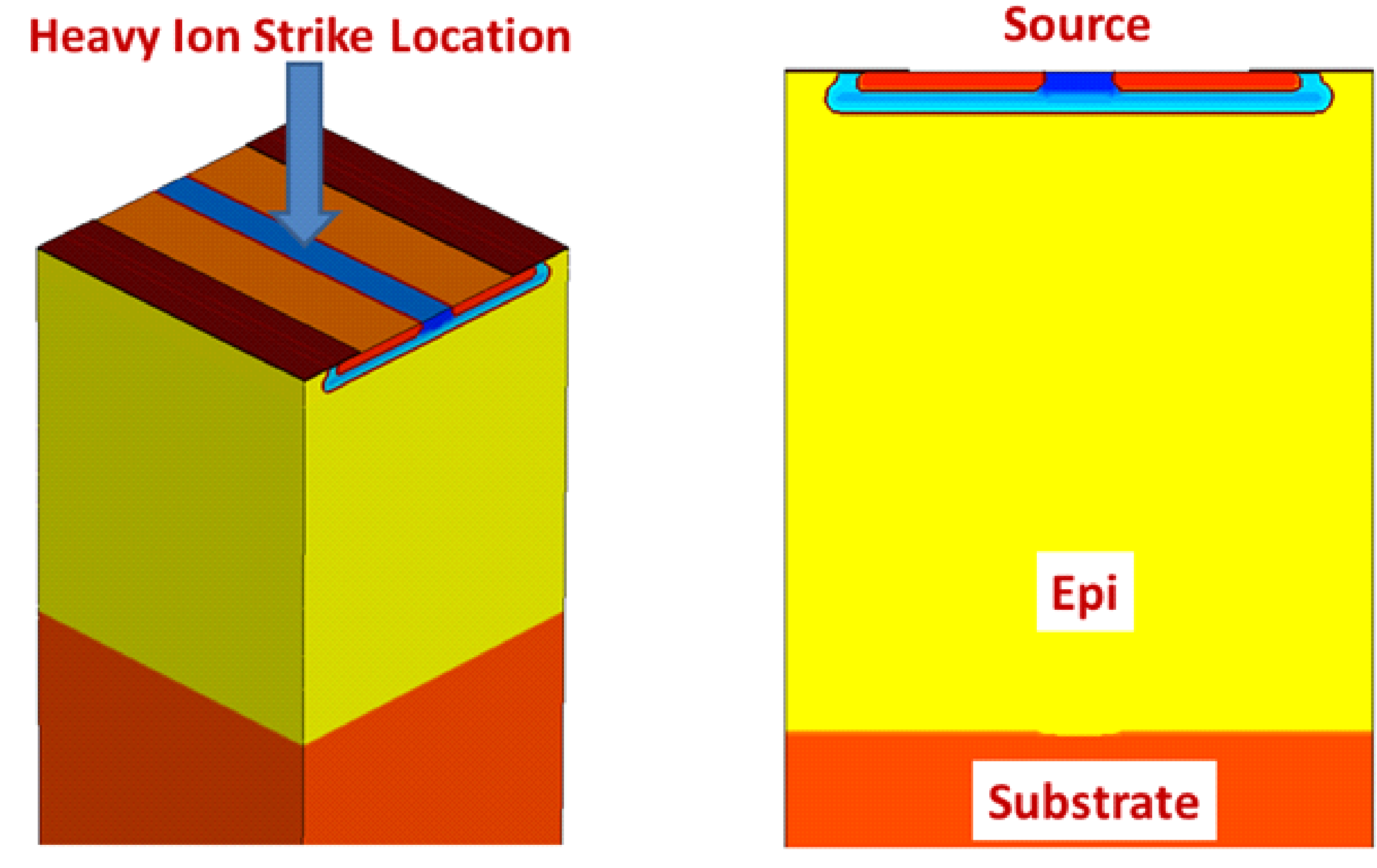

\section{SEB Simulation}

-Silver ion strike at a Linear Energy Transfer (LET) of $46 \mathrm{MeV}-\mathrm{cm}^{2} / \mathrm{mg}$ in the center of the source contact region

- Burnout is assumed when the temperature reaches $3000 \mathrm{~K}$ anywhere

- Device fails between 475 and $500 \mathrm{~V}$

$\checkmark$ e-h plasma formation from heavy ion strike

$\diamond$ Transit of e-h carriers to modulate electric field causing an electric field peak that persists at the epi/substrate interface

$\checkmark$ Enhanced electric field causes significant impact ionization and localized heating at the epi/substrate interface until thermal destruction

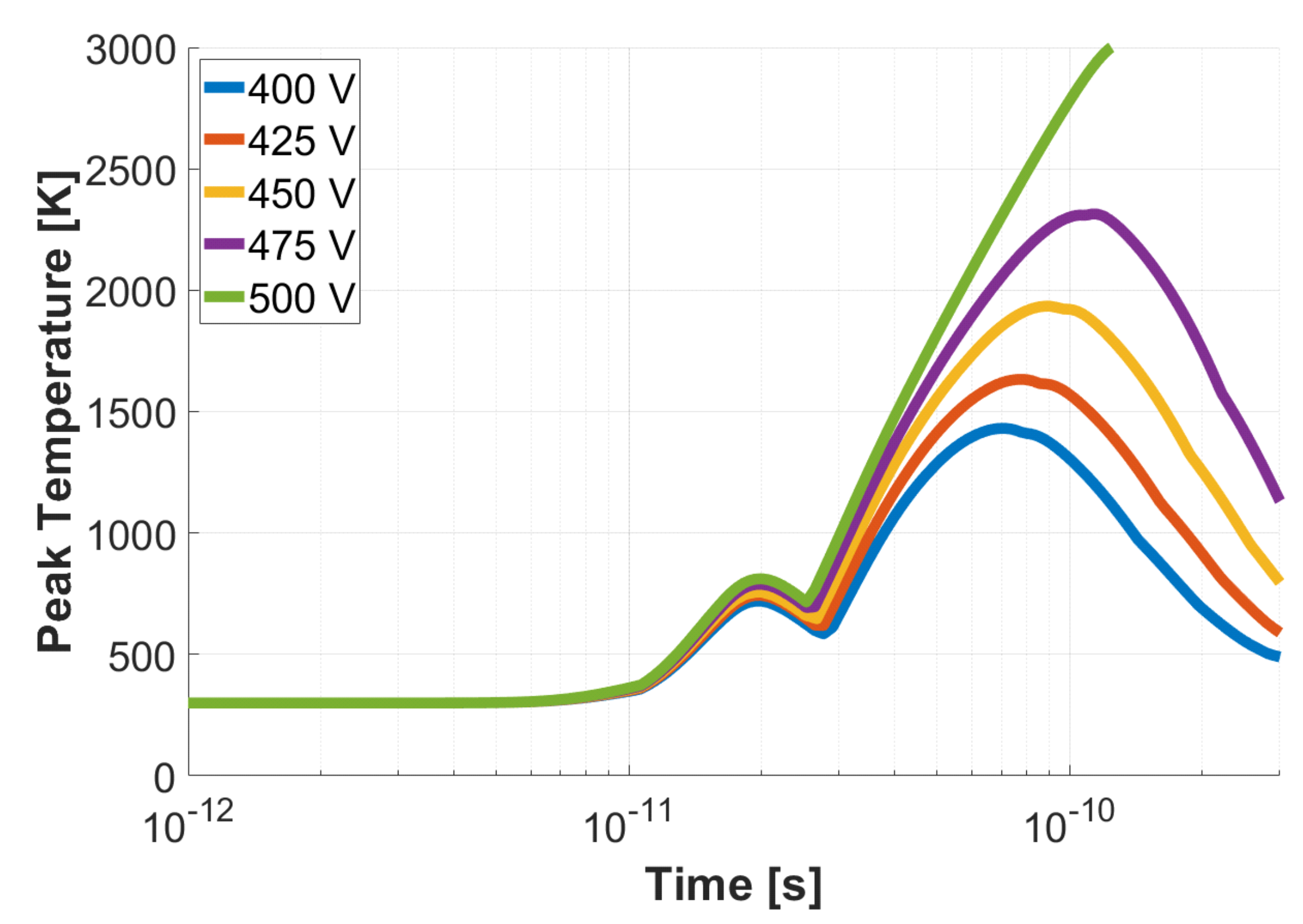

Along heavy ion track

2-D

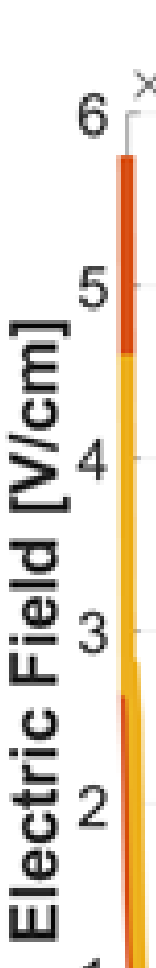
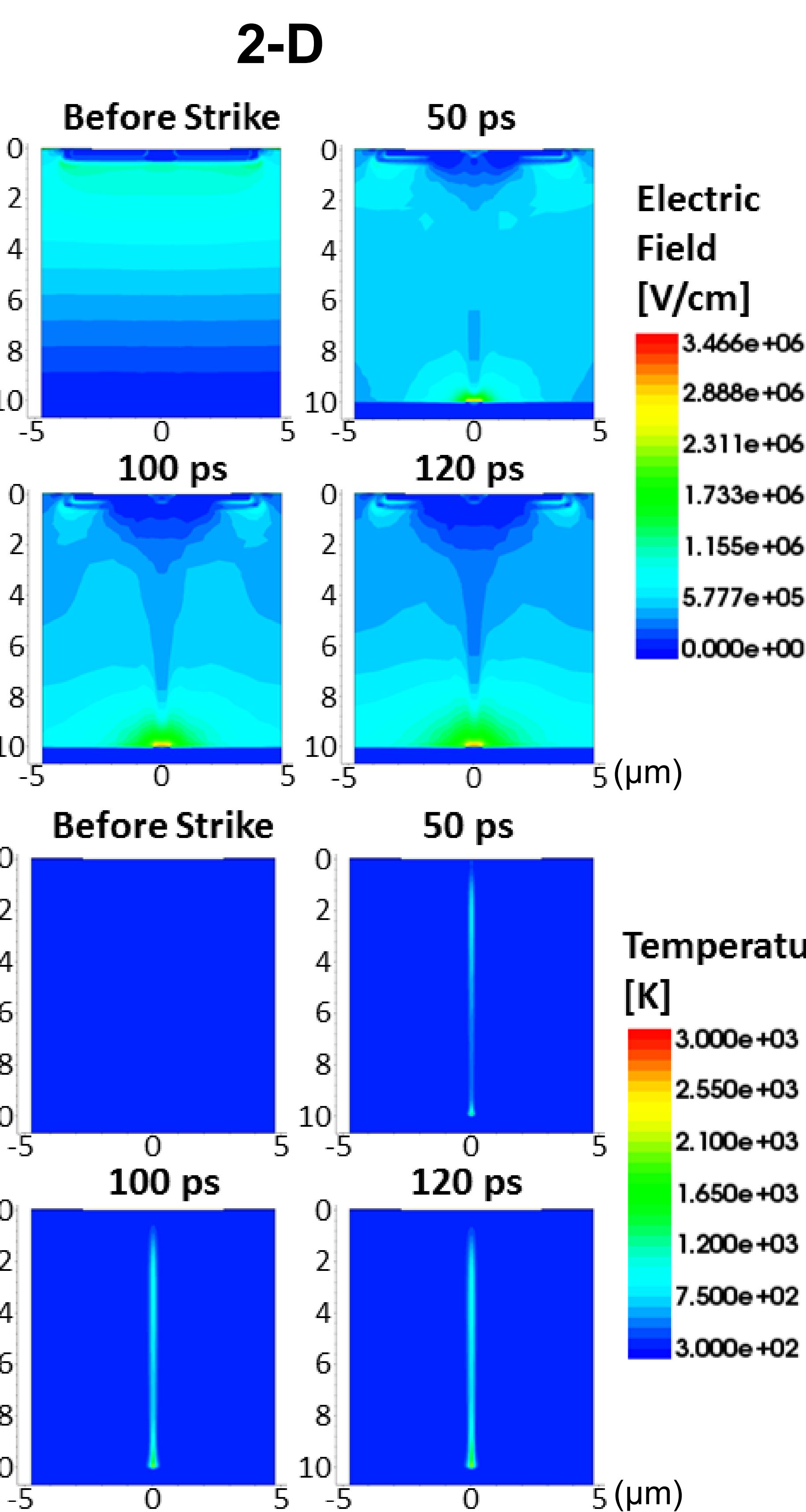

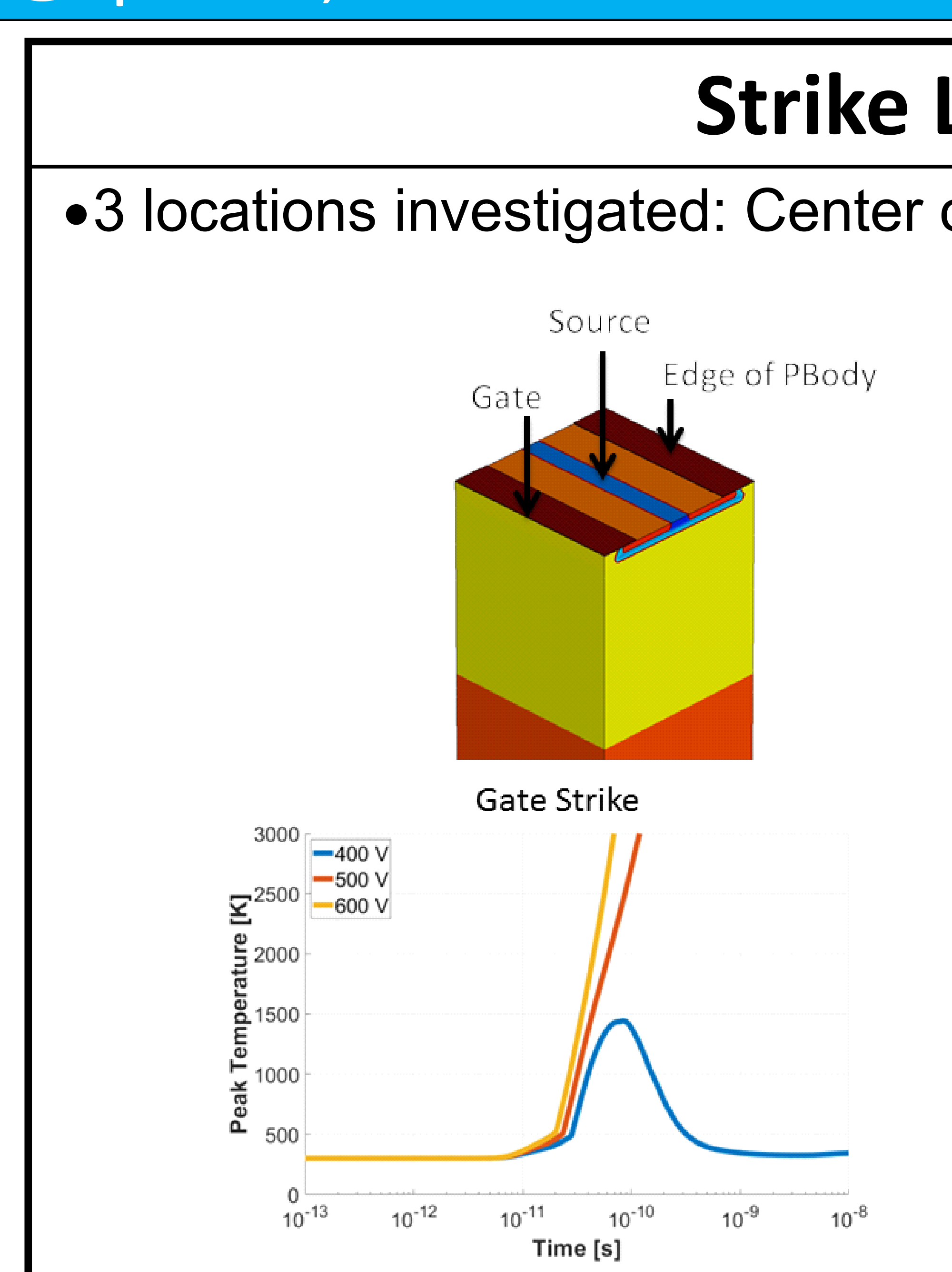

Strike Locations

-No dependence found between strike location and SEB

\begin{tabular}{|l|}
\multicolumn{1}{c|}{ Improved Designs } \\
\hline -2 $\mu \mathrm{m}$ additional, $\mathrm{N}$-type layer inserted in between the original epi lay- \\
er and substrate \\
- SEB threshold voltage was raised to over $900 \mathrm{~V}$ \\
- Electric field enhancement at epi/substrate interface has been sup- \\
pressed \\
- Failure of device is due to excessive heating from an accumulation of
\end{tabular}
carriers at a depth of 3 to $4 \mu \mathrm{m}$ below top surface

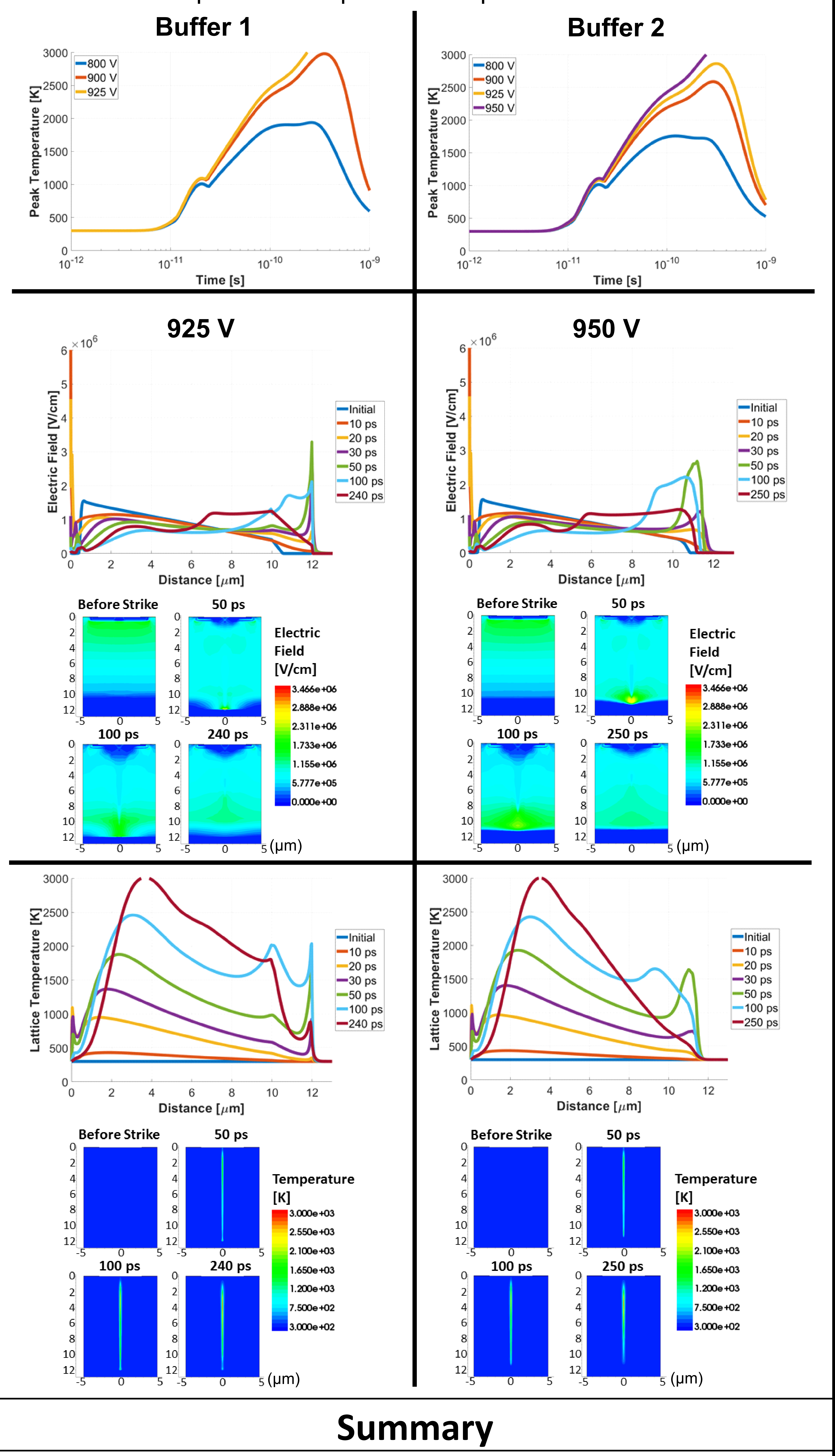

- Failure of commercial design is due to the electric field at the epi/ substrate interface is high enough to cause significant impact ionization, which in turn causes significant heating and eventual failure

- Creation of two buffer designs were investigated and allowed for the device to operate at over $900 \mathrm{~V}$ before SEB occurred and this is due to the reduction of the electric field at the epi/substrate interface

\section{References}

- T. Nitta et al., in PCIM Europe 2016; International Exhibition and Conference Power berg, Germany, 566 .

.D. J. Lichtenwalner et al., Material Science Forum 924, 559 (2018).

• A. F. Witulski et al., IEEE Trans. Nucl. Sci. 65, 256 (2018).

-X. Zhu et al., ICSCRM (2017).

- S.-H. Ryu et al., Material Science Forum 717, 1059 (2012).

- T. Shoji, S. Nishida, K. Hamada, and H. Tadano, Japanese J. Appl. Phys. 53,

This work was supported by an Early Stage Innovations grant (NNX17AD05G) from NASA's Space Technology Research Grants Program. 